of the field and directed towards this center, the scalar function $g(|\mathbf{r}|)$ defined in (15) is expressed by

$$
g(|\mathbf{r}|)=-K|\mathbf{r}|^{-2},
$$

where $K$ is a constant. The force $F$, with which this field acts on a body $V$, bounded by a closed surface $A$, may be found by substituting (25) in (19). We hereby find

$$
\mathbf{F}=-\frac{K}{2} \int_{A}[\mathbf{e} \cos (\mathbf{n}, \varrho)-\mathbf{n}]|\mathbf{r}|^{-1} d a .
$$

For demonstrating the application of this formula we consider a sphere with center $P$ and radius $R$ in a central field of force of the type discussed here. The center $\mathrm{O}$ of the field is assumed to be situated on the surface of the sphere. The previously used Fig. 2 may also be applied as an illustration of the present problem. For reasons of symmetry the force will be parallel with the radius $P O$ to the center of the field; in computing the surface integral we therefore retain only the component in this direction of the differential contributions. We find from (26)

$\mathrm{F}=-\frac{K}{2} \int_{0}^{\pi} \mathrm{t}\left[\cos \frac{\theta}{2} \cos \frac{\theta}{2}-\cos \theta\right]\left[2 R \cos \frac{\theta}{2}\right]^{-1} 2 \pi R \sin \theta R d \theta=-\frac{4 \pi}{3} K R \mathrm{t}$,

where the first factor $\cos \theta / 2$ stands for $\cos (\boldsymbol{o}, t)$, the second factor $\cos \theta / 2$ for $\cos$ $(\mathbf{n}, \mathfrak{\varrho}), \cos \theta$ for $\cos (\mathbf{n}, \mathbf{t}),[2 R \cos \theta / 2]^{-1}$ for $|\mathbf{r}|^{-1}$ and $2 \pi R \sin \theta R d \theta$ for $d a$.

From the theory for Newtonian potentials ${ }^{3}$ it is known that the force $\mathbf{F}$ from the field of force discussed here may be computed by assuming that the mass of the sphere is concentrated to its center. As the volume of the sphere is $4 \pi / 3 R^{3}$ and the distance between the center of the sphere and the center of the field $R$, we therefore find the force $\mathbf{F}$ expressed as

$$
\mathbf{F}=-\left(K R^{-2}\right)\left(\frac{4 \pi}{3} R^{3}\right) \mathbf{t}
$$

This expression is seen to be identical with the expression (27), found by using the surface integral method. The result from the potential theory used above was derived by carrying out a double integration. The calculation has consequently been simplified also in the present case by the use of the vector formula (14).

${ }^{3}$ O. D. Kellogg, Foundations of potential theory, Berlin, 1929.

\title{
THE CIRCULAR PLATE WITH ECCENTRIC HOLE*
}

\section{By SAMUEL D. CONTE (Wayne University)}

1. Introduction. It is well known that the equation governing the small deflections of a thin uniform plate, assumed homogeneous and isotropic, is

$$
D \Delta \Delta w+P(x, y)=0,
$$

*Received March 20, 1951. 
where $P(x, y)$ is an arbitrary load per unit area acting normal to the upper surface, $D=2 d^{3} / 3\left(1-\sigma^{2}\right)$ is the flexural rigidity of the plate, $d$ is the thickness of the plate, and $\Delta$ the Laplacian operator in two dimensions. All quantities appearing in this and in all succeeding equations are in dimensionless form. It is assumed that there are no forces acting on the edges of the plate other than the reactions due to the method of fixing of the plate. The state of stress and strain at every point of the plate can be completely determined when the deflection $w$ of points originally on the middle plane of the plate is known. Complete solutions of the plate equation (1) under arbitrary normal loads and for various boundary conditions are known for thin rectangular [1], circular [2], or elliptic [3] plates. It is proposed in this paper to present a solution of this problem when the plate is bounded by two circles, one of which is interior to and eccentric to the other.

2. Bipolar coordinates. The boundary conditions are considerably simplified when bipolar coordinates $(\alpha, \beta)$ are introduced. The conformal transformation

$$
z=x+i y=c \tanh \frac{1}{2}(\alpha+i \beta)=c \tanh \frac{1}{2} \zeta,
$$

maps the rectangular region $\left[\alpha_{1} \leqq \alpha \leqq a_{0},-\pi \leqq \beta \leqq \pi\right]$ in the $\zeta$-plane onto the region between two eccentric circles in the $z$-plane. As shown in Fig. 1, the boundary of this region consists of the exterior circle $\alpha=\alpha_{1}$ and the interior circle $\alpha=\alpha_{0}$, with $\alpha_{0}>\alpha_{1}$.

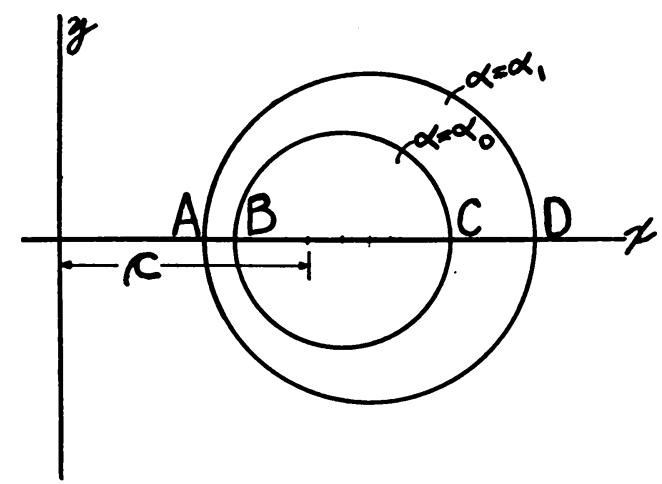

FIG. 1.

The constant $c$ is the pole of the system. The straight line segments $A B$ and $C D$ are given respectively by $\beta=0$ and by $\beta=\pi$. Under the transformation (2) the Laplacian operator becomes

$$
\Delta=h^{2}\left(\frac{\partial^{2}}{\partial \alpha^{2}}+\frac{\partial^{2}}{\partial \beta^{2}}\right),
$$

where $(c h)^{2}=(\cosh \alpha+\cos \beta)^{2}$ is the Jacobian of the transformation. If $(h w)$ is taken as the dependent variable instead of $w$, the harmonic and the biharmonic equations become respectively,

$$
\begin{aligned}
\Delta w & =\left\{\operatorname{ch}\left(\frac{\partial^{2}}{\partial \alpha^{2}}+\frac{\partial^{2}}{\partial \beta^{2}}\right)-2 \sinh \alpha \frac{\partial}{\partial \alpha}+2 \sin \beta \frac{\partial}{\partial \beta}+\cosh \alpha-\cos \beta\right\}(h w)=0, \\
\Delta \Delta w & =h^{3}\left(\frac{\partial^{4}}{\partial \alpha^{4}}+\frac{2 \partial^{4}}{\partial \alpha^{2} \partial \beta^{2}}+\frac{\partial^{4}}{\partial \beta^{4}}-2 \frac{\partial^{2}}{\partial \alpha^{2}}+2 \frac{\partial^{2}}{\partial \beta^{2}}+1\right)(h w)=0 .
\end{aligned}
$$


Thus the biharmonic equation becomes a linear partial differential equation with constant coefficients [4]. Separation of variables now yields as solutions the following series of biharmonic functions which have the period $2 \pi$ in $\beta$;

$$
h w=\sum_{n=0}^{\infty} \phi_{n}(\alpha) \cos n \beta+\psi_{n}(\alpha) \sin n \beta,
$$

where $\phi_{0}(\alpha)=A_{0} \sinh \alpha+B_{0} \alpha \sinh \alpha+D_{0} \cosh \alpha+E_{0} \alpha \cosh \alpha$,

$$
\begin{aligned}
& \psi_{0}(\alpha)=0, \\
& \phi_{1}(\alpha)=A_{1}+B_{1} \alpha+D_{1} \cosh 2 \alpha+E_{1} \sinh 2 \alpha, \\
& \psi_{1}(\alpha)=A_{1}^{\prime}+B_{1}^{\prime} \alpha+D_{1}^{\prime} \cosh 2 \alpha+E_{1}^{\prime} \sinh 2 \alpha, \\
& \phi_{n}(\alpha)=A_{n} \cosh (n+1) \alpha+B_{n} \cosh (n-1) \alpha+D_{n} \sinh (n+1) \alpha \\
& +E_{n} \sinh (n-1) \alpha, \\
& \psi_{n}(\alpha)=A_{n}^{\prime} \cosh (n+1) \alpha+B_{n}^{\prime} \cosh (n-1) \alpha+D_{n}^{\prime} \sinh (n+1) \alpha \\
& +E_{n}^{\prime} \sinh (n-1) \alpha,
\end{aligned}
$$

and $A_{n}, B_{n}, D_{n}, E_{n}, A_{n}^{\prime}, B_{n}^{\prime}, D_{n}^{\prime}, E_{n}^{\prime}(n=0,1, \cdots)$ are eight sets of arbitrary constants.

3. Method of solution. It is now proposed to obtain a solution of the boundary value problem consisting of Eq. (1) and one of the usual boundary conditions. The most common of these boundary conditions are those corresponding to clamped edges and to simply supported edges. For a clamped edge the boundary conditions in terms of $(h w)$ are

$$
h w=\frac{\partial(h w)}{\partial \alpha}=0 .
$$

For a simply supported edge the boundary conditions are

$$
\begin{gathered}
(h w)=0, \\
\left\{\cosh \alpha\left(\frac{\partial^{2}}{\partial \alpha^{2}}+\sigma \frac{\partial^{2}}{\partial \beta^{2}}\right)-(1+\sigma) \sinh \alpha \frac{\partial}{\partial \alpha}+(1+\sigma) \sin \beta \frac{\partial}{\partial \beta}\right. \\
+\sigma \cosh \alpha-\cos \beta\}(h w)=0 .
\end{gathered}
$$

A solution of the boundary value problem consisting of Eq. (1) and conditions (5) or (6) is sought in the form

$$
h w=w_{0}+w_{1},
$$

where $w_{0}$ is the series of biharmonic functions (4) and $w_{1} / h$ is a particular solution of the plate equation. If $w_{1}$ is expanded into a Fourier series, boundary conditions (5) or (6) give rise to a system of eight equations which can be solved for the eight sets of unknowns 
which appear in $w_{0}$. If, for example, a clamped plate is subjected to a uniform normal load $p$, a particular solution of the resulting boundary value problem is

$$
\frac{w_{1}}{h}=\frac{A}{h^{2}}, \quad A=-\frac{p c^{2}}{16 D},
$$

and since the deflection must be an even function of $\beta$, the biharmonic function is taken as

$$
w_{0}=\sum_{n=0}^{\infty} \phi_{n}(\alpha) \cos n \beta .
$$

If $w_{1}$ is expanded into a Fourier series, the boundary conditions yield four sets of equations for the coefficients $A_{n}, B_{n}, D_{n}, E_{n}$ which appear in $\phi_{n}(\alpha)$. Explicit expressions for these coefficients are presented in the author's thesis, and it is shown there that the series (9) with these coefficients converges absolutely and uniformly in $\alpha$ and $\beta$ over the domain between the eccentric circles.

4. Particular solution for an arbitrary analytic load. Consider first a load function which is homogeneous of degree $n$ in $x$ and $y$. Expressed in bipolar coordinates such a load has the form

$$
P(\alpha, \beta)=\sum_{k \neq 0}^{n} \alpha_{n k} \frac{\sinh ^{n-k} \alpha \sin ^{k} \beta}{h^{n}} .
$$

A particular solution of the plate equation (1) for this load function is given by

$$
\frac{w_{1}}{h}=\sum_{k=0}^{n} A_{n k} \frac{\sinh ^{n-k+2} \alpha \sin ^{k+2} \beta}{h^{n+4}},
$$

where the coefficients $A_{n k}$ are determined by the following system of equations

$$
\begin{aligned}
& 2(n-k+2)(n-k+1)(k+2)(k+1) A_{n k} \\
& \quad+(n-k+4)(n-k+3)(n-k+2)(n-k+1) A_{n(k-2)} \\
& \quad+(k+4)(k+3)(k+2)(k+1) A_{n(k+2)}=Q_{n k}, \quad(k=0,1, \cdots, n) .
\end{aligned}
$$

This system of $(n+1)$ equations can be solved uniquely for the $(n+1)$ unknowns $A_{n k}$ $(k=0,1, \cdots, n)$. These results for $k=2 m, k=2 m+1$ are:

$$
\begin{gathered}
A_{n(2 m)}=\sum_{r=0}^{m-1}(-1)^{r} \frac{(n-2 m+2 r+2) \cdots(n-2 m+3)(r+1)}{(2 m+2) \cdots(2 m-2 r-1)} \\
\cdot\left\{1-\frac{(m+1)(r+[n / 2]-m+2)}{(r+1)([n / 2]+2)}\right\} Q_{n(2 m-2 r-2)} \\
+\sum_{r=0}^{[n / 2]-m}(-1)^{r+m+[n / 2]} \frac{(n+2) \cdots(n-2 m+3)}{(2[n / 2]+4)(2 m+1) !} \\
\cdot \frac{(r+1)\left(2 r+\epsilon_{1}^{n}\right)}{(n+2) \cdots(2[n / 2]-2 r+1)} \cdot Q_{n(2[n / 2]-2 r)},
\end{gathered}
$$




$$
\begin{aligned}
& A_{n(2 m+1)}=\sum_{r=0}^{[(n+1) / 2-1]}(-1)^{r} \frac{(n-2 m+2 r+1) \cdots(n-2 m+2)(r+1)}{(2 m+3) \cdots(2 n-2 r)} \\
& \cdot\left\{1-\frac{(m+1)(r+[(n+1) / 2]-m+1)}{([(n+1) / 2]+1)(r+1)}\right\} a_{n(2 m-2 r-1)} \\
& +\sum_{r=0}^{[n / 2]-m+\epsilon_{3}^{n}}(-1)^{r+m+[n / 2]-\epsilon_{3}^{n}} \frac{(n+1) \cdots(n-2 m+2)(m+1)}{([(n+1) / 2]+1)(2 m+3) !} \\
& \cdot \frac{(r+1)\left(2 r+\epsilon_{2}^{n}\right) !}{(n+1) \cdots(2[(n+1) / 2]-2 r)} \cdot a_{n(2 !(n+1) / 2]-2 r-1)} .
\end{aligned}
$$

In these equations [ $n / 2]$, for example, means the largest integer in $n / 2$, and the symbol $\epsilon_{m}^{n}$ has the following meaning: $\epsilon_{m}^{n}=0$ if $(n+m)$ is odd; $\epsilon_{m}^{n}=1$ if $(n+m)$ is even. All factors in these equations must appear in descending order. When this is not the case, as for example, when $r=0$, the entire factor is to be replaced by unity.

Consider now an analytic load function $P(x, y)$. If its Taylor's series is rearranged in homogeneous powers of degree $n$ in $x$ and $y$ together and expressed in bipolar coordinates, it has the form

$$
P(\alpha, \beta)=\sum_{n=0}^{\infty} \sum_{k=0}^{n} a_{n k} \frac{\sinh ^{n-k} \alpha \sin ^{k} \beta}{h^{n}}
$$

where $\mathcal{Q}_{n k}$ are known constants. By means of the method shown above a particular solution of the plate equation (1) is given formally by

$$
\frac{w_{1}}{h}=\sum_{n=0}^{\infty} \sum_{k=0}^{n} A_{n k} \frac{\sinh ^{n-k+2} \alpha \sin ^{k+2} \beta}{h^{n+4}} .
$$

The coefficients $A_{n k}$ for each $n$ and $k$ can be determined by means of Eqs. (13), (14).

5. Special loads. When the load function has certain specialized forms, the peculiar properties of the operator $\Delta$ in bipolar coordinates may be profitably exploited to yield comparatively simple solutions of the boundary value problems in thin plate theory. It may be proved by induction or by direct calculation that if $u_{n}=\sinh n \alpha / h^{n}$, or if $u_{n}=\cosh n \alpha / h^{n}$, then

$$
\Delta u_{n}=\frac{2 n^{2}}{c} u_{n-1} ;
$$

and if $v_{n}=\cos n \beta / h^{n}$ or if $v_{n}=\sin n \beta / h^{n}$, then

$$
\Delta v_{n}=\frac{-2 n^{2}}{c} v_{n-1}
$$

Thus, if the load function is any finite or infinite sum of the functions $u_{n}$ or $v_{n}$, this stepping-down property of the operator $\Delta$ may be utilized to obtain a particular solution of the plate equation. A complete solution satisfying boundary conditions (5) or (6) may then be obtained by following the procedure given in Section 3 .

As another special case let the load function be

$$
P(\alpha, \beta)=k h^{3} \sinh p \alpha \cos q \beta,
$$


where $k$ is a known constant and $p, q$ are integers but $p \neq(q \pm 1)$. If the plate is clamped at the edges, a particular solution of (1) is readily obtained in the form

$$
w_{1}=A \sinh p \alpha \cos q \beta,
$$

where

$$
A=-\frac{k}{D} \frac{1}{\left(p^{2}-q^{2}\right)^{2}-2 q^{2}-2 p^{2}+1} .
$$

The boundary conditions may be satisfied by taking only those biharmonic terms involving $\cos q \beta$. Hence a complete closed form solution for a clamped plate under the load (17) is given by

$$
h w=A \sinh p \alpha \cos q \beta+\phi_{q}(\alpha) \cos q \beta,
$$

where $\phi_{q}=A_{q} \cosh (q+1) \alpha+B_{q} \cosh (q-1) \alpha+D_{q} \sinh (q+1) \alpha+E_{q} \sinh (q-1) \alpha$. The boundary conditions (5) yield four equations which determine uniquely the four constants $A_{a}, B_{a}, D_{a}, E_{a}$. Indeed, a solution in closed form can always be obtained if the normal load is any finite sum of terms of the following forms: $h^{3} \cosh p \alpha \cos q \beta, h^{3} \cosh p \alpha$ $\sin q \beta, h^{3} \sinh p \alpha \cos q \beta, h^{3} \sinh p \alpha \sin q \beta$. If $p=(q \pm 1)$ in any of these loads the function is itself biharmonic. If, for example,

$$
P(\alpha, \beta)=k h^{3} \sinh (q+1) \alpha \sin q \beta,
$$

a particular solution of the plate equation which is periodic in $\beta$ is given by

$$
w_{1}=A \alpha \cosh (q+1) \alpha \sin q \beta
$$

with

$$
A=-\frac{k}{D} \cdot \frac{1}{2 q(q+1)(q+4)} .
$$

A closed form solution involving only four of the biharmonic functions can again be obtained as in the previous case. We note, however, that if the plate is simply supported at the edges, no finite number of biharmonic terms will suffice to yield a solution of the resulting boundary value problem under loads of the form (17) or (18).

\section{BibLIOGRAPHY}

1. S. Timoshenko, Theory of plates and shells, McGraw-Hill, New York, 1940.

2. S. Jen, Bending of circular plates, Thesis, University of Michigan, 1948.

3. C. Perry, Elliptic plates, Thesis, University of Michigan, 1949.

4. G. B. Jeffery, Plane stress and plane strain in bipolar coordinates, Phil. Trans. Roy. Soc., London (A) 221, 265 (1921).

5. S. D. Conte, Thin plate problems in bipolar coordinates, Thesis, University of Michigan, 1950. 